\title{
Total Electron Content (TEC) estimation over Pakistan from local GPS network using spherical harmonics
}

\author{
Maria Mehmood ${ }^{*}, 1$, Sajid Saleem ${ }^{1}$, Renato Filjar $^{2}$, Najam Abbas Naqvi ${ }^{3}$, Arslan Ahmed ${ }^{4}$ \\ (1) National University of Science and Technology (NUST), Islamabad, Pakistan \\ (2) Independent satellite navigation and spatial statistical learning advisor and Professor of Electronic Engineering at \\ Zagreb University of Applied Sciences, Zagreb, Croatia and faculty of engineering University of Rijeka, Croatia \\ (3) Institute of Space Technology (IST), Islamabad, Pakistan \\ (4) Sukkur IBA University, Sukkur, Pakistan
}

Article history: received February 21, 2020; accepted June 1, 2020

\begin{abstract}
Many organizations allow GNSS users to access Global Ionosphere Maps (GIMS). However, the TEC estimates derived from GIMs are of insufficient quality to describe small-scale TEC variations over Pakistan. In this paper, the first local TEC map over Pakistan for the year 2019, derived from a regional GPS network, is presented. Spherical harmonics expansion is employed to estimate TEC with the spatial resolution of latitude $0.2^{\circ}$ x longitude $0.2^{\circ}$ and temporal resolution of 5 minutes. The impact of changing the degree/order of harmonics is assessed and it is determined that harmonic expansion up to 6 degrees is sufficient for estimating accurate TEC map for the region of interest. We have demonstrated that the TEC maps of Pakistan generated by local model conform better to the GIM by Center of Orbit Determination (CODE) $(R M S=5.83)$ as compared to International Reference Ionosphere (IRI-2016) $(R M S=7.18)$. We found that the TEC estimated by the local model shows a better correlation to measured TEC; CODE-GIM overestimated TEC, while IRI-2016 underestimates it. Moreover, it was observed that TEC peaks during noon (1100-0100 LT) and Equinox (April). The residuals of local TEC estimates with respect to TEC obtained from CODEGIM indicate the inaccuracy of CODE-GIM over the region of Pakistan: highest deviation of TEC from local model with respect to CODE -GIM was observed in April $(R M S=8.73)$ and minimum in October $(R M S=2.78)$. We have also analyzed the performance of our maps in geomagnetically disturbed days. The research presented in this paper will contribute towards the ionosphere study over Pakistan, where limited research is available currently.
\end{abstract}

Keywords: GPS; Total Electron Content; Ionosphere; Pakistan; Spherical Harmonics.

\section{Introduction}

Total Electron Content (TEC) from Global Navigation Satellite System (GNSS) has a number of significant applications in a variety of fields including but not limited to navigation, timing, surveying, agriculture, 


\section{Maria Mehmood et al.}

telecommunications, financial industry and military. As with all electromagnetic signals, GNSS signals are also prone to a number of natural and artificial error sources such as atmospheric errors, clock errors, multipath, hardware biases, jamming and spoofing. However, studies have confirmed the GNSS ionospheric delay as the single most influential cause of GNSS positioning error [Klobuchar, 1982; Feltens and Schaer, 1998; Krankowski et al. 2005; Perez, 2017; Liu et al., 2018]. Therefore, it becomes pertinent to model the ionosphere delay and variations and account for this error in positioning.

The ionosphere is a region of Earth's atmosphere containing charged particles along with free electrons having boundary limits starting from $\sim 50 \mathrm{~km}$ and extending more than $\sim 1000 \mathrm{~km}$. This region causes a frequencydependent propagation effect on the RF signals passing through it, such as phase delay and carrier advance. One of the parameters to quantify the ionosphere is TEC which is the total number of free electrons present in a square meter cross sectional area between the satellite and the receiver.

The analysis of the received GNSS signal properties may contribute towards detection and identification of the ionospheric condition variations due to space weather events such as solar flares, solar radio bursts (SRBs) and coronal mass ejections (CME) [Cannon, 2013]. With the deployment of several GNSS stations around the globe, it has become an alternate source of data to model and monitor the ionosphere TEC. Moreover, the vast network of GNSS stations across the globe allows large scale spatial coverage thus resulting in a database enough to create global maps of TEC variability [Mendillo, 2006]. In fact, several international organizations use the GNSS data to provide global ionosphere maps (GIMs) representing the TEC distribution using below various techniques.

International GNSS Service (IGS) uses a weighted mean of other analysis centers to create a GIM in Bernese software [Liu et al., 2018]. Jet propulsion laboratories (JPL) uses a three shell model based on spline functions. This approach uses the Kalman filter and 4DVar (four-dimensional variation) to estimate the state vectors and update the observation matrix [JPL, 2015]. CODE uses carrier-smoothed pseudo ranges in solar-geomagnetic reference frames and spherical harmonic expansion up to 15 degrees. European Space Agency (ESA) uses spherical harmonic functions expansion up to 15 degrees and assumes that the ionosphere is concentrated as a single-layer at $450 \mathrm{~km}$. Technical University of Catalonia (UPC) uses a tomographic approach which involves feeding the GPS NTrip data streams from a global network of receivers to solve the main tomographic equation with the help of TOMION software:

$$
L_{1}=S+B_{1} \cong N_{e, i} \Delta l_{i}
$$

where $S$ is the STEC, B is the unknown ambiguity, $N_{e, i}$ is the mean electron density within each voxel and $\Delta l_{i}$ is the length intersection between each voxel.

These institutes estimate GIMs with a resolution of $5^{\circ}$ in latitude, $2.5^{\circ}$ in longitude and 2 hours in time [Hernández-Pajares et al., 2009]. However, the resolution of global TEC map is insufficient for derivation of the regional TEC map of required quality [Wielgosz et al., 2003]. On the other hand the empirical models, like IRI, are not efficient in describing certain ionosphere anomalies especially those that only occur locally.

A lot of effort has been made to enhance the knowledge of ionosphere variability at regional level by generation of local/regional TEC maps. The techniques for mapping TEC are mainly divided in to two categories: (1) Functionbased models and (2) Grid-based models. The former includes mathematical models such as spherical harmonics and B-splines while the latter approach divides the geographic region into different grids and interpolates data over each grid with no IPP. The regional TEC map over Canada has been developed using spherical harmonics from daily RINEX files recorded at an interval of 30 secs [Reza et al., 2011]. The regional TEC model over Africa referred to as SATCP (South African TEC prediction) has been developed using spherical harmonic technique which generates the model over Africa with a resolution of 1 minute and produces results comparable to that of ionosondes [Habarulema et al., 2011]. Neural Networks specifically multilayer perceptron (MLPs) and radial basis function networks (RBFN), have been used to demonstrate the TEC mapping over Turkey due to their non-linear modeling capability [Yilmaz et a., 2009]. The TEC variations over Japanese islands have been mapped with $0.5^{\circ} \mathrm{x} 0.5^{\circ}$ grid using spherical harmonics expanded to as high as 60 degrees [Ping et al., 2002]. A recent study [Krypiak-Gregorczyk et al., 2017] used a novel approach utilizing thin splines to model the ionosphere TEC over Europe. The TEC over the region of China has been developed using tomography [Zhizhao and Gao, 2004] as well as spherical harmonics [Zhao et al., 2016]. Studies have also been carried out in Japan for estimating the TEC using spherical harmonics with GEONET (GNSS Earth Observation network) data [Ohashi et al., 2015]. 
The standard ionosphere models (e.g IRI and GIM) have poor performance in the equatorial or low latitude regions as well as regions with sparse data; which is why GNSS users in these regions cannot fully rely on standard models. This creates the need to have tools that will enable the local GNSS users with reliable TEC data. In this context, a local TEC model describing the variation of TEC for year 2019 is presented. The local TEC map has been generated with a spatial resolution of latitude $0.2^{\circ}$ x longitude $0.2^{\circ}$ and temporal resolution of 5 minutes, using spherical harmonic expansion up to 6 degrees. The focus of the research is not only to demonstrate the performance of standard models over Pakistan but to present an improved alternative in the form of locally-driven TEC maps. These TEC maps provide an accurate assessment of the TEC variation over Pakistan for year 2019 as compared to the currently-used CODE-GIM. The main reason for this is because among all the IGS (International GNSS Service) stations used to generate the global map, not even one is located in Pakistan (Figure 1). The results from the generated model are compared to CODE-GIM as well as IRI-2016 to determine the improvement offered in terms of TEC estimation.

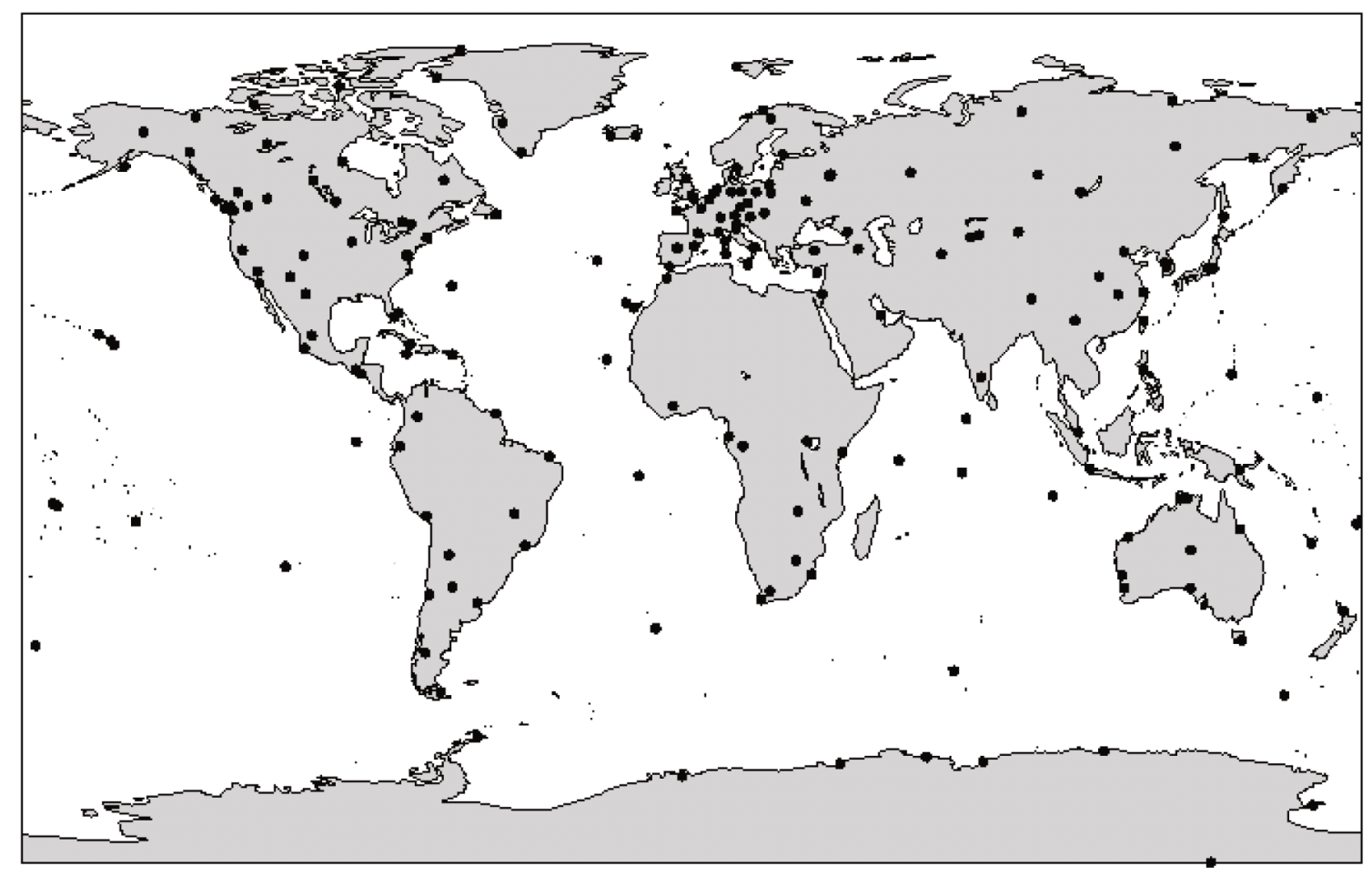

Figure 1. GPS tracking stations used for CODE-GIM.

\section{Methodology}

\subsection{Data set}

The data set used for this research is collected from four GNSS stations located in Pakistan (Figure 2). The selection of reference sites is such that the obtained Ionosphere Pierce Points (IPPs) cover the whole region of interest. Moreover, the monitoring equipment selection, installation and operation methods were in complaint with the guidelines provided by IGS [IGS, 2015]. The lack of IPPs over extreme south-west reveals that it will be advantageous to add another station towards the south-west of Pakistan in the future. The data is archived in RINEX format on daily basis with an interval of 30 seconds. The quality of logged data is ensured by passing it through TEQC software developed by UNAVCO, available at https://www.unavco.org/software/data-processing/teqc/teqc.html. 


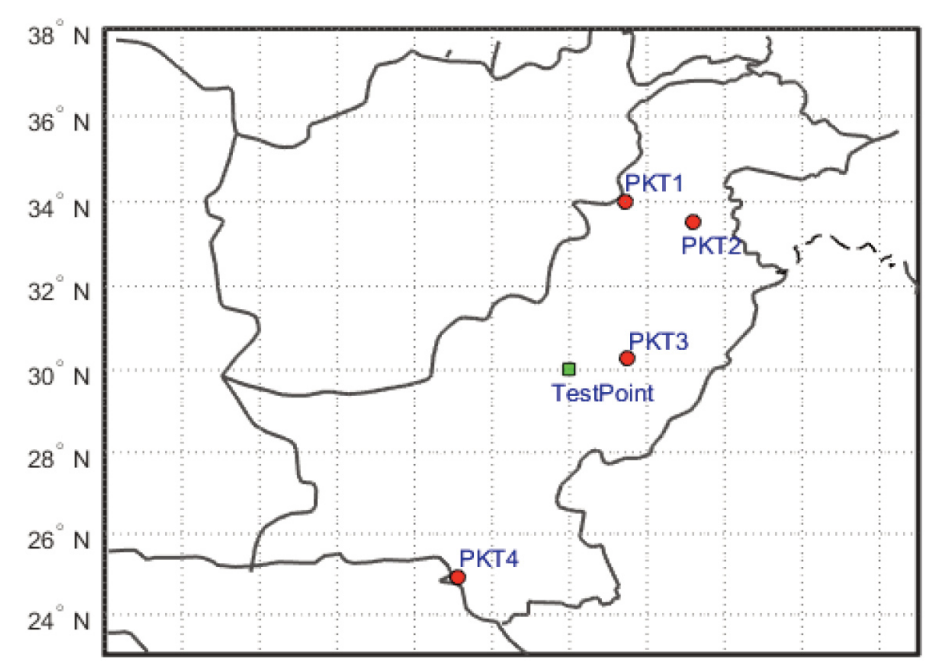

Figure 2. Location of GNSS stations in Pakistan used in this study (red) and location of test point (green) used for model validation.

\subsection{TEC estimation from GPS}

The RINEX file from a dual frequency GPS receiver includes carrier phase $\left(\Phi_{1}\right.$ and $\left.\Phi_{2}\right)$ as well as pseudorange $\left(P_{1}\right.$ and $\left.P_{2}\right)$ values for both frequencies, $f_{1}(1575.41 \mathrm{MHz})$ and $f_{2}(1227.60 \mathrm{MHz})$. These observations are defined as [Barrile, Cacciola, \& Cotroneo, 2006; Sharifi \& Farzaneh, 2017]:

$$
\begin{gathered}
P_{i}=\rho+c .(d t-d T)+d_{o}+d_{T}+I_{i}+b_{P i}^{s}+b_{P i}^{r}+\varepsilon_{P i} \\
\Phi_{i}=\rho+c .(d t-d T)+d_{o}+d_{T}-I_{i}+b_{\Phi i}^{s}+b_{\Phi i}^{r}+\varepsilon_{\Phi i}+\lambda_{i} N_{i}
\end{gathered}
$$

Where $i$ indicates the frequency, $f_{1}$ or $f_{2}, \rho$ is the geometric range between the satellite and receiver in meters, $d_{o}$ is the orbital error in meters, $d t$ are $d T$ the satellite clock error and receiver clock error with respect to GPS time in seconds, $d T$ is the troposphere error in meters, $I$ is the ionosphere delay for each frequency $b^{s}$ and $b^{r}$ are the satellite delay and receiver delays in meters for both carrier phase and pseudorange on each frequency, $\varepsilon_{i}$ are the measurement noise errors in meters, $N_{i}$ are the integer ambiguities and $c$ is the speed of light $\left(3.08 \times 10^{8} \mathrm{~m} / \mathrm{s}\right)$. The geometry-free linear combination of pseudo range is then obtained by estimating the difference between the pseudo ranges on both frequencies, as shown below:

$$
P_{4}+P_{1}-P_{2}=\left(I_{1} I_{2}\right)+b_{P i}^{S}-b_{P i}^{r}
$$

The geometry-free combination solution for TEC estimation yields the slant ionospheric TEC (STEC) which is expressed as:

$$
P_{4}=40.3 \operatorname{STEC}\left(\frac{f_{2}^{2}-f_{1}^{2}}{f_{1} f_{2}}\right)+b_{P i}^{S}-b_{P i}^{r}
$$

TEC is measured in terms of TECU where 1 TECU $=10^{16}$ electrons $/ \mathrm{m} 2$. The same procedure may be followed to obtain the TEC from carrier-phase measurements. In case of two-dimensional ionospheric modelling the STEC is converted to vertical TEC (VTEC) using the single layer model (SLM) (Figure 3).

SLM assumes that the ionosphere is concentrated as a single layer around 350-450 km above the Earth surface. STEC is mapped on a vertical to obtain VTEC using an obliquity function at the ionosphere pierce point (IPP) defined 


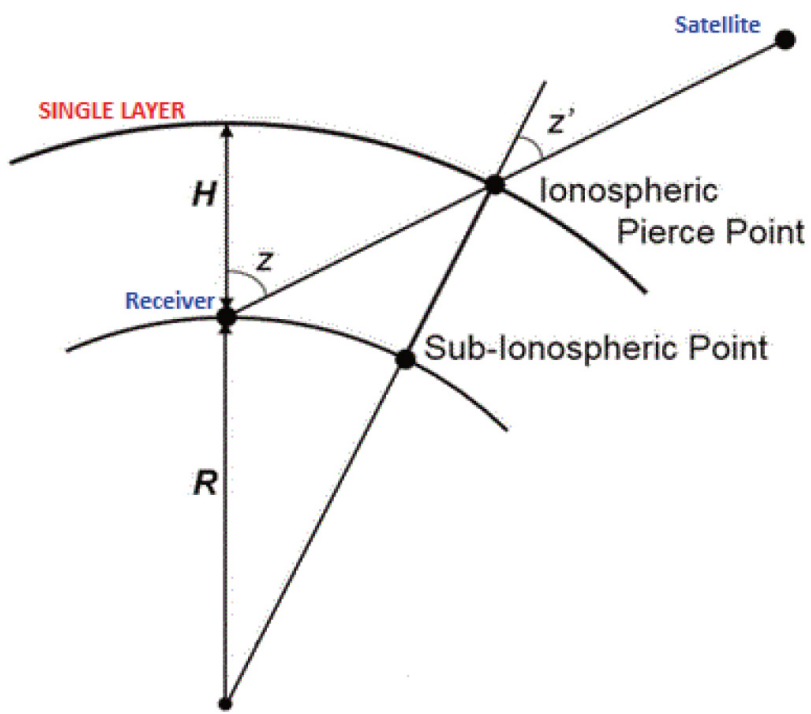

Figure 3. SLM for conversion of STEC to VTEC.

by [Han et al., 2013]. The VTEC is expressed as:

$$
V T E C=\cos \left[\arcsin \left(\frac{R}{R+H} \sin z\right)\right] S T E C
$$

where $\mathrm{R}$ is the equatorial radius of the earth $(6378 \mathrm{~km}), \mathrm{H}$ the altitude of the ionosphere shell and $\mathrm{z}$ the zenith angle [Davies, 1990]. For this study the STEC was mapped onto VTEC using SLM with a height of $350 \mathrm{~km}$.

The spherical harmonic expansion is applied to VTEC as described in [Zhao et al., 2016]:

$$
\operatorname{VTEC}(\theta, \xi)=\sum_{k=0}^{k=\max } \sum_{m=0}^{k}\left[\widetilde{P}_{n_{k}}^{m}(\cos \theta) \cdot\left(A_{k}^{m} \cdot \cos (m, \xi)\right)+B_{k}^{m} \cdot \sin (m, \xi)\right]
$$

where $A_{k}$ and $B_{k}$ are the unknown model co-efficients. $\theta$ and $\xi$ are the magnetic latitude and local time ionosphere pierce points respectively in the spherical cap harmonic coordinate system, which are obtained from the measured longitude and latitude of ionosphere pierce points. The degree and order of spherical harmonics are represented by $n$ and $m$ and signify the resolution in latitude and longitude respectively. $\widetilde{P}_{n_{k}}{ }^{m}$ is the normalized associated Legendre function and maybe expressed as $\widetilde{P}_{n_{k}}^{m}=\Lambda(\mathrm{n}, \mathrm{m}) P_{m n}$ where $\Lambda$ refers to the normalization function being applied on the un-normalized polynomial $P_{m n}$. The normalization function may be expanded as:

$$
\Lambda=\sqrt{2 \frac{2 n+1(n-m) !}{1+\delta_{0 m}(n+m) !}}
$$

where $\delta$ is the Kronecker Delta. Substituting the equation 5 \& 6 into 7, results in:

$$
\sum_{k=0}^{k=m a x} \sum_{m=0}^{k}\left[\widetilde{P}_{n_{k}}^{m}(\cos \theta) \cdot\left(A_{k}^{m} \cdot \cos (m, \xi)\right)+B_{k}^{m} \cdot \sin (m, \xi)\right]=\cos \left[\arcsin \left(\frac{R}{R+H} \sin z\right)\right] \cdot\left[-\frac{f_{1} f_{2}}{f_{2}^{2}-f_{1}^{2}} P_{4}-b_{P i}^{s}-b_{P i}^{r}\right]
$$

The four unknown parameters: $A_{k}^{m}, B_{k}^{m}, b_{P i}^{s}$ and $b_{P i}^{r}$, are estimated using least square (LS) method. An external constraint is assumed that the sum of all GPS satellite DCBs is zero. This is known as the zero-mean constraint and separates the receiver DCBs from satellite DCBs as well as makes Eq. 9 full rank. Further details of this method of DCB estimation may be obtained at [Jin et al., 2012]. 


\section{Maria Mehmood et al.}

\subsection{TEC estimation from CODE}

The CODE of IGS analysis center is operated by the Astronomical Institute of the University of Bern (AIUB), Switzerland. CODE uses data from more than 200 GNSS stations (Figure 1) and uploads daily Global Ionosphere Maps (GIM) on their FTP http://ftp.aiub.ch/CODE/IONO/. The ionosphere data is disseminated in the form of TEC values in IONEX [Hernández-Pajares et al., 2009] or GTEX [Saito et al., 2017] format. A detailed description of these products is available on their website at http://cmslive3.unibe.ch/unibe/philnat/aiub/content/e15/ e59/e440/e447/e458/index_eng.html. The TEC data in the IONEX files is sorted according to the geographical latitude and longitude. The latitudes range from $-180^{\circ}$ to $180^{\circ}$ with a step size of $5^{\circ}$ and the longitude range from $-87.5^{\circ}$ to $87.5^{\circ}$ with a step size of $2.5^{\circ}$; this makes a total of 5183 grid points (each with a unique estimated TEC value) in a CODE GIM. These values are then interpolated to estimate TEC values all over the globe.

However, since there are no grid points (or IGS stations) with in Pakistan (Figure 1) any TEC estimation over this region using CODE-GIM will have significant interpolation error. Poor spatial coverage and large deviations from a regional model, where less or no local GNSS data was used were also observed by [Ohashi et al., 2015]. For this study the IONEX files for the year 2019 were download from the aforementioned website and interpolated over the region of interest via post-processing.

\subsection{TEC estimation from IRI-2016}

The International Reference Ionosphere (IRI) model has been developed by Committee on Space Research (COSPAR) and the International Union of Radio Science (URSI) in 1960. The model has been progressing ever since by inclusion of better modelling techniques and has been updated by release of new editions including IRI-78 [Rawer et al., 1978], IRI-85 [Bilitza, 1986], IRI-1990 [Bilitza, 1990], IRI-2000 [Bilitza, 2001], and IRI-2007 [Bilitza and Reinisch, 2008].

The IRI model divides the ionosphere into six sub-regions and provides information on several ionosphere parameters including electron density and temperature, ion composition, drift and temperature and TEC up to a height of $2000 \mathrm{~km}$. The IRI model is the most-widely used empirical and deterministic model to observe ionosphere trend for space weather. The advantage of this model is that since it is empirical, it is independent of our changing knowledge about the physics of atmosphere. The disadvantage lies in the fact that its accuracy depends on its database, so the geographical and temporal locations not covered by the database will experience error. The database of IRI is concentrated in mid latitudes, therefore low-latitude regions experience error [Bilitza, 2018].

The latest IRI model is IRI-2016 and a detailed description is available at http://irimodel.org/. The TEC estimates from IRI-2016 can be accessed online from the website https://ccmc.gsfc.nasa.gov/modelweb/models/ iri2016_vitmo.php and the software available at http://irimodel.org/IRI-2016/. For this study the hourly TEC values were obtained at the region of interest for year 2019 via aforementioned website. The following settings were used: NeQuick for Ne Topside and URSI for Ne F-peak and the storm model was set on.

\section{Results and discussion}

This study presents a local TEC map for Pakistan $\left(20^{\circ}-40^{\circ}\right.$ latitude, $55^{\circ}-80^{\circ}$ latitude), as shown in Figure 2 , for year 2019, using spherical harmonic functions. The local map provides a resolution of $0.2^{\circ} \times 0.2^{\circ} \times 5$ mins and is estimated at a height of $350 \mathrm{~km}$. For global representation of TEC the degree and order are set higher than 10 for generation of global map with sufficient resolution, but for regional mapping they need to be determined accordingly [Zhao et al., 2016]. For this study the spherical harmonic expansion of several degrees was examined starting from 3 and going upwards. The expansion of up to 6 satisfies the previously set requirements for the experimental local TEC map accuracy over Pakistan, since there is no significant change in RMS error with respect to CODE-GIM after further expansion (Table 1). 


\begin{tabular}{clllll} 
Time & \multicolumn{5}{c}{ Degree } \\
& $\mathbf{3}$ & $\mathbf{4}$ & $\mathbf{5}$ & $\mathbf{6}$ & $\mathbf{7}$ \\
\hline 06:00 UT & 12.605 & 10.536 & 10.420 & 10.501 & 10.500 \\
\hline 12:00 UT & 9.605 & 5.660 & 5.632 & 5.630 & 5.625 \\
\hline 18:00 UT & 9.561 & 6.442 & 6.420 & 6.412 & 6.401 \\
\hline 24:00 UT & 5.937 & 5.102 & 5.084 & 5.004 & 5.080 \\
\hline
\end{tabular}

Table 1. RMS of error between TEC estimated from local model and CODE-GIM for different hours and different degrees of spherical harmonic expansion.

A comparative analysis for DOY: 045, 2019 (1100 LT) is presented (Figure 4) as an example. The top panel (Figure 4) show the TEC maps generated by CODE-GIM, this study and IRI-2016 respectively. The CODE-GIM and IRI-2016 have been interpolated to a grid of latitude $0.2^{\circ}$ x longitude $0.2^{\circ}$ for the sake of comparison. It can be observed that all maps show a similar variation but the local TEC map presents more clear and defined features. The bottom panel (Figure 4) present the RMS between this study and CODE-GIM, CODE-GIM and IRI-2016 and this study and IRI-2016 respectively. It is observed the TEC values estimated by local map are in a better agreement with those by CODEGIM as compared to those by IRI-2016; Moreover, the difference between this study and CODE-GIM is lesser than that between CODE-GIM and IRI-2016. Similar results were observed by [Xin et al., 2016] for their regional ionosphere model over China. The reason for a higher RMS error for IRI-2016 might be because the IRI-2016 database mainly comprises of TEC values from mid-latitude regions [Sur and Paul, 2013]. Moreover the inaccurate calculation of the topside profiling also leads to under/estimation of TEC values. We believe that our maps conform better to CODE-GIMs since both are based on TEC derived from a network of GNSS stations rather than an empirical model like IRI-2016.

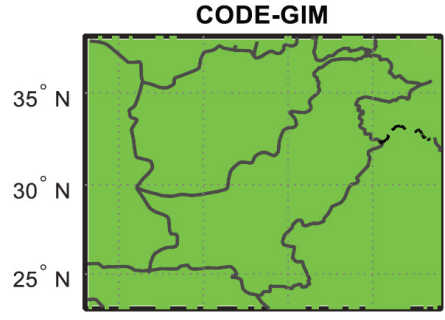

$60^{\circ} \mathrm{E} \quad 65^{\circ} \mathrm{E} \quad 70^{\circ} \mathrm{E} 75^{\circ} \mathrm{E}$ CODE-Local

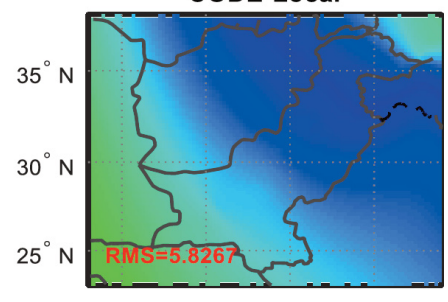

$60^{\circ} \mathrm{E} \quad 65^{\circ} \mathrm{E} 70^{\circ} \mathrm{E} 75^{\circ} \mathrm{E}$

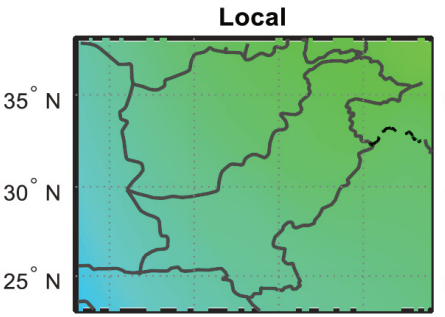

$60^{\circ} \mathrm{E} \quad 65^{\circ} \mathrm{E} \quad 70^{\circ} \mathrm{E} \quad 75^{\circ} \mathrm{E}$ CODE-IRI2016

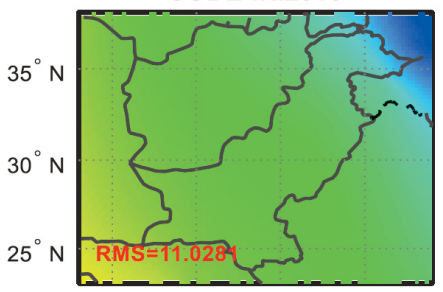

$60^{\circ} \mathrm{E} \quad 65^{\circ} \mathrm{E} \quad 70^{\circ} \mathrm{E} \quad 75^{\circ} \mathrm{E}$

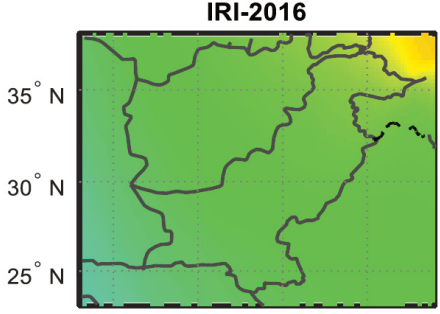

$60^{\circ}$ E $\quad 65^{\circ}$ E $\quad 70^{\circ}$ E $75^{\circ} \mathrm{E}$ Local-IRI2016

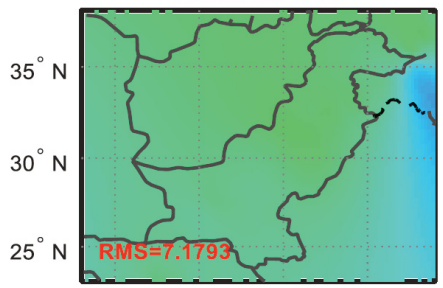

$60^{\circ} \mathrm{E} \quad 65^{\circ} \mathrm{E} \quad 70^{\circ} \mathrm{E} \quad 75^{\circ} \mathrm{E}$
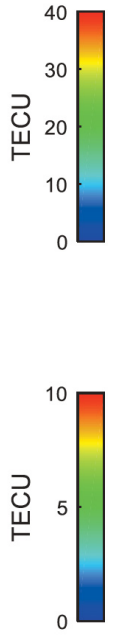

Figure 4. TEC maps generated from CODE-GIM, Local model and IRI-2016 (top panel) and their differences (bottom panel) for DOY: 045,2019 at 1100 LT. 


\section{Maria Mehmood et al.}

The same strategy was extended to obtain the residuals between CODE-This study and IRI-This study for every hour of each day of the year 2019. The histogram of the residuals (Figure 5), with extreme outliers ignored, reveals that the residuals between CODE and this study are positive with a mean error of 7.26 and variance of 3.31 ; whereas the residuals of IRI and this study were negative with a mean of -11.99 and variance of 2.41 . The residual analysis confirms that the results obtained from this study conform better to CODE GIM rather than IRI-2016 and that CODE GIM overestimates the TEC whereas IRI-2016 underestimates it.
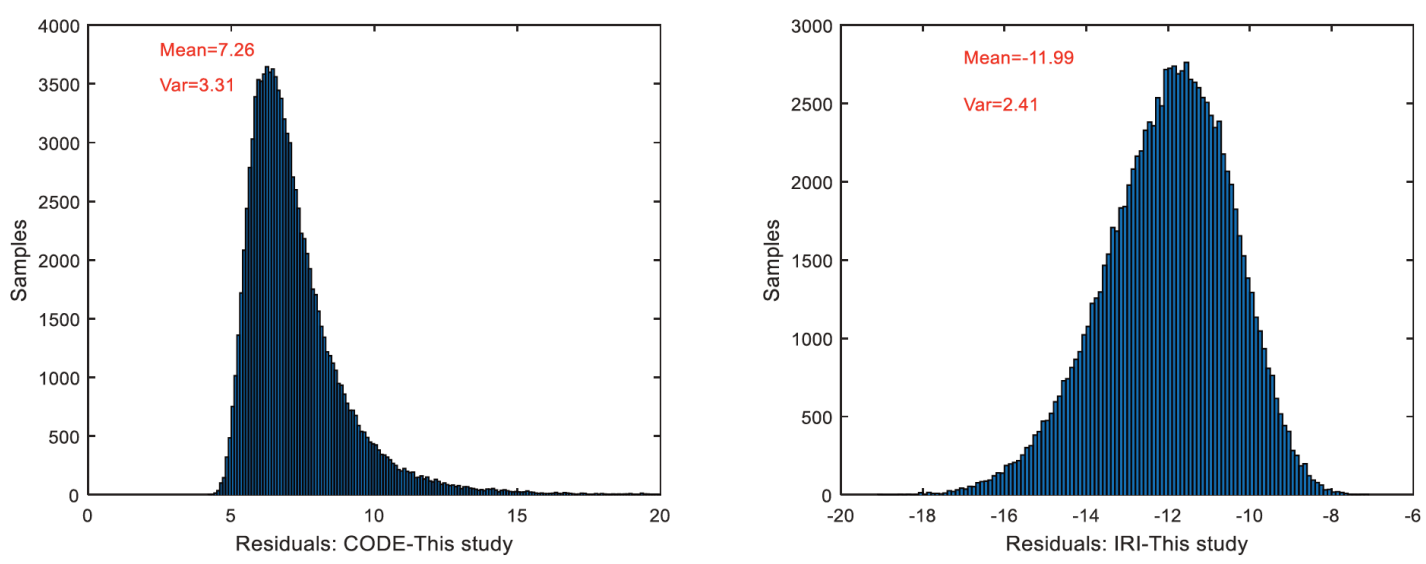

Figure 5. Residual Analysis for residuals between CODE-This study and IRI-This study for year 2019.

In order to validate the model with actual data, a GPS scintillation receiver was set up at a test location of latitude $30^{\circ} \mathrm{E}$, longitude $70^{\circ} \mathrm{N}$ for one month (June, 2019) to record the TEC estimates. This location was not used in the generation of local map and the measured TEC values from the scintillation receiver at this location were correlated to the TEC values estimated by local TEC map, CODE-GIM and IRI-2016 (Figure 6). It was observed that the TEC estimated by this study showed a higher correlation to the actual recorded TEC as compared to CODE-GIM and IRI2016. Therefore, the trend of TEC depicted by this study conforms better to the actual TEC; CODE-GIM overestimates TEC values by 20-25 TECU whereas IRI-2016 underestimates TEC values by 5-10 TECU. A possible reason of underestimation is because the mid-latitude TEC, which is used for IRI database, has a significantly lower value as compared to low latitude regions.
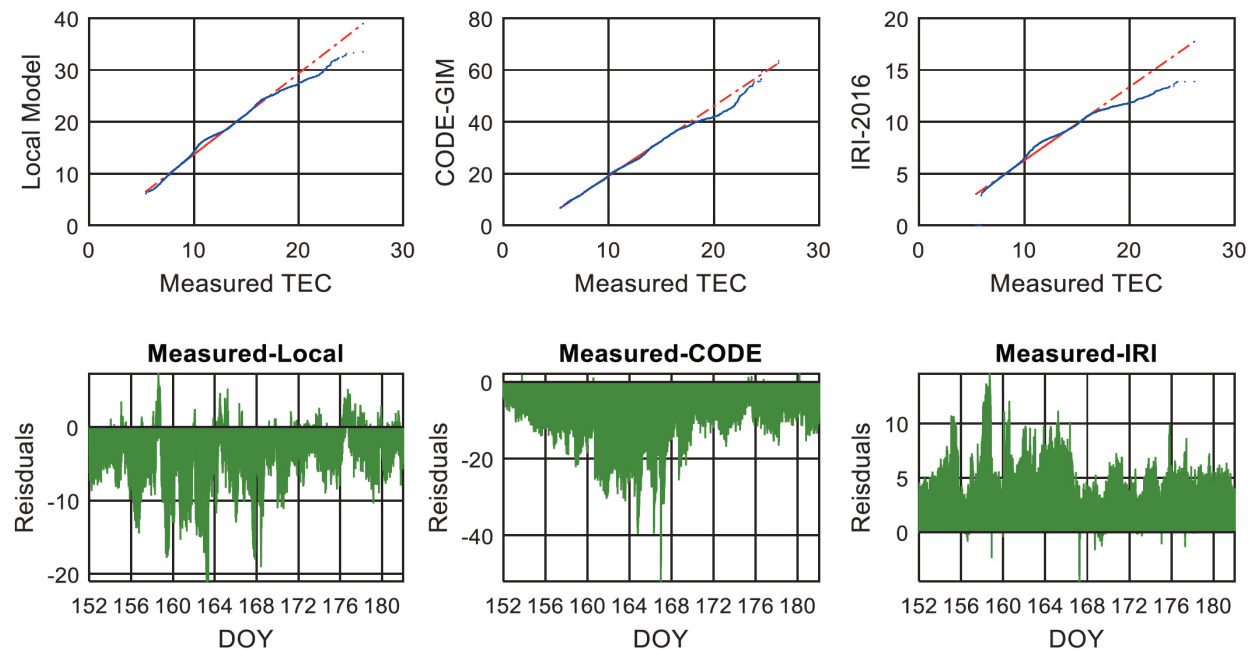

Figure 6. Correlation between Measured TEC by scintillation receiver and TEC estimated from Local Model, CODE-GIM and IRI-2016 (top panel) and residuals of TEC estimated from the three models with measured TEC (bottom panel). 
For further analysis the interpolated data estimated by CODE-GIM and this study is obtained at the aforementioned test location for the whole year 2019. The year is divided according to three seasons: Equinox (March, April, September, October), Summer Solstice (May, June, July, August) and Winter Solstice (January, February, November, December). The hourly mean of TEC from both models is estimated by taking the average of all quiet days $(\mathrm{Kp}<4)$ of each season (Figure 7). The TEC residuals at each hour is calculated by taking the difference between the two hourly means of both models at same hour thus:

$$
d T E C=M e a n_{-} T E C_{-} C O D E_{j}-M e a n_{-} T E C_{-} L_{o c a l}
$$

where $j=$ hour number .

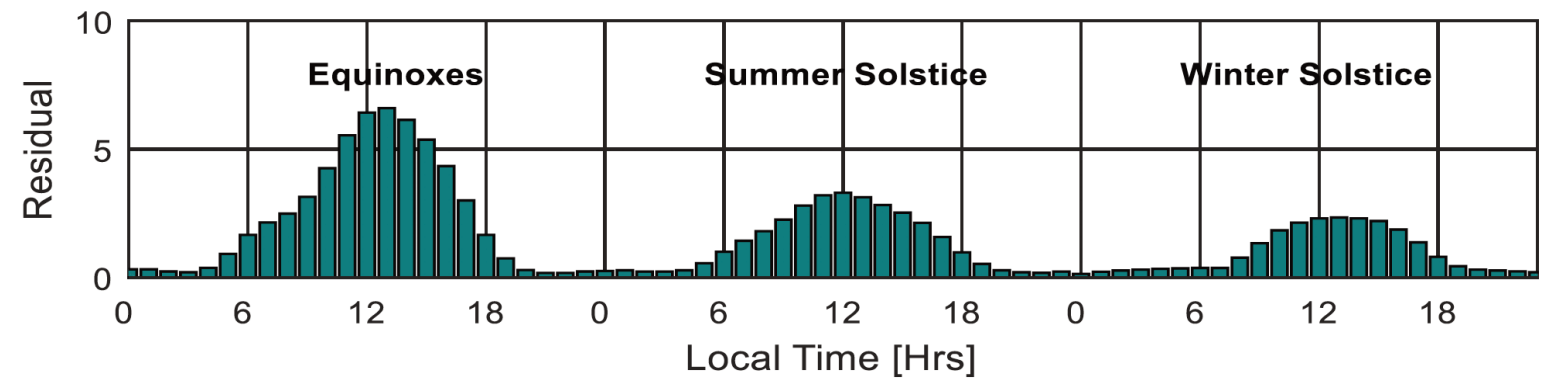

Figure 7. Seasonal Variation in TEC residuals of TEC estimated from local model and CODE-GIM at test point.

It can be observed from Figure 7, that the residuals are higher during the day as compared to night and maximum during Equinoxes. Furthermore, the daily mean of TEC estimated by this study and CODE-GIM at the test location was observed and their difference was calculated (Figure 8). The residual TEC was then divided into months and a monthly RMS of the residual TEC was calculated. A maximum RMS of 8.736 TECU was recorded in the Equinox (April) and minimum of 2.780 TECU was obtained during winter solstice (October).
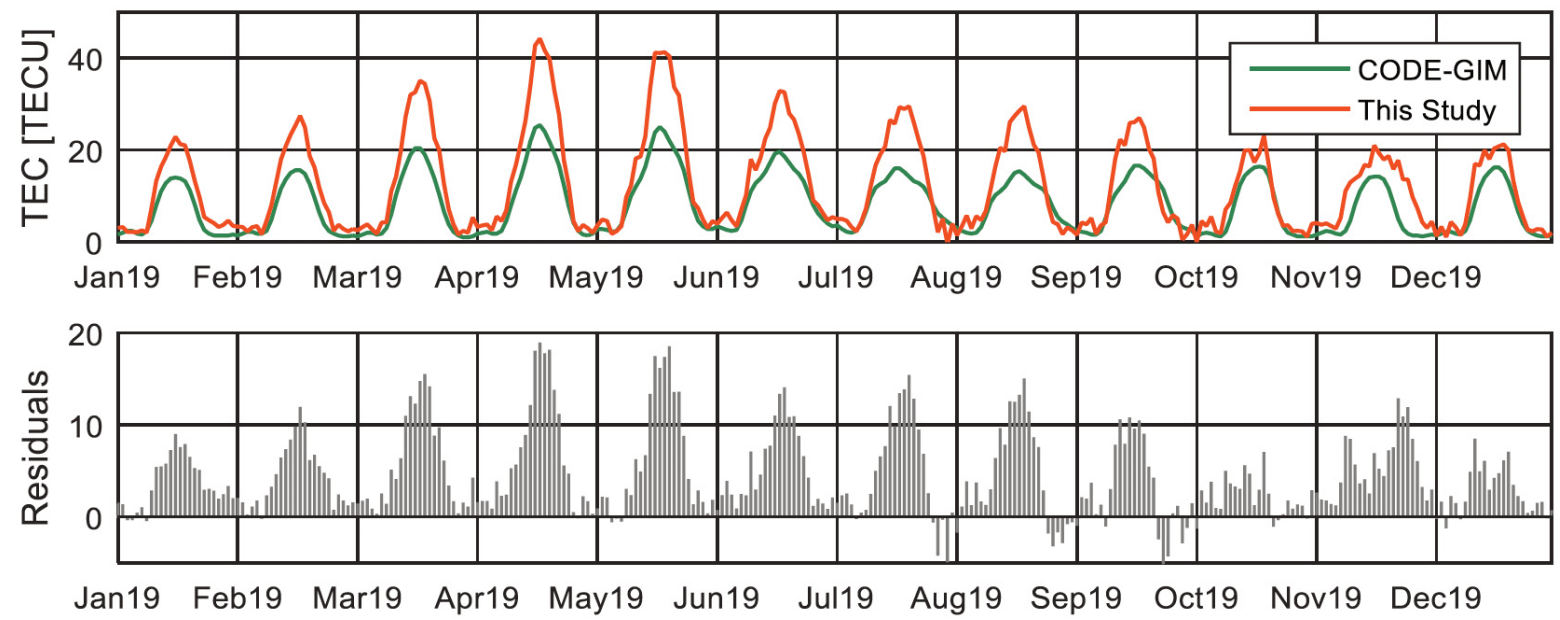

Figure 8. TEC variation at test point (30 deg latitude, 70 deg longitude) estimated by CODE-GIM and Local Model for year 2019. 


\section{Maria Mehmood et al.}

The VTEC maps were generated using this study over 24 hours for DOY 312 (Year 2019) to assess the diurnal variation (Figure 9). It can be see that VTEC values increase slowly in the morning with sunrise and decrease with sunset in the evening. The maxima is obtained around noon (1100 LT-0100 LT), which may be due to the intensification of photoionization process as described in [Chapman, 1931]. A similar pattern was observed by previous studies over the region where VTEC was estimated using GPS and IRI (International Reference Ionosphere) [Tariq et al., 2019].
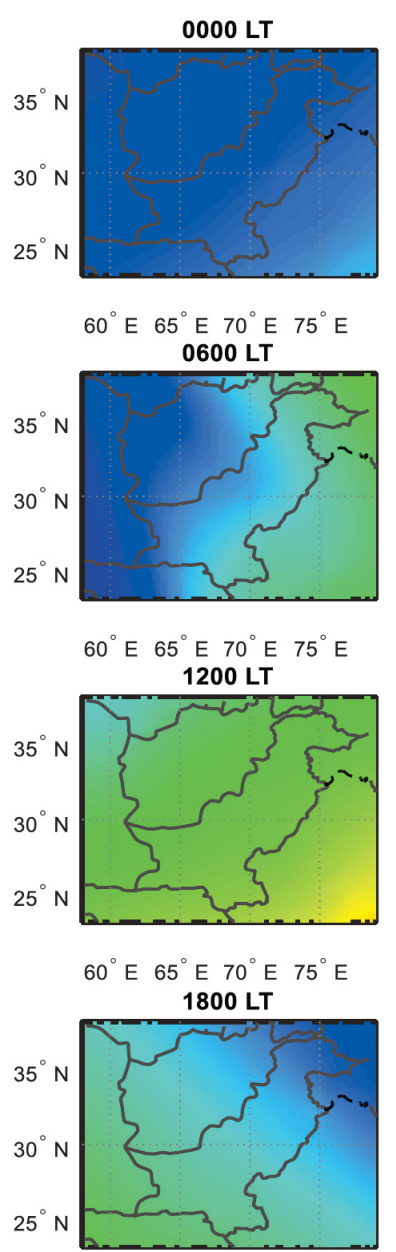

$60^{\circ}$ E $65^{\circ}$ E $70^{\circ}$ E $75^{\circ} \mathrm{E}$

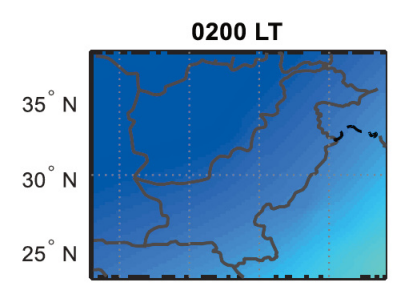

$60^{\circ} \mathrm{E} 65^{\circ} \mathrm{E} 70^{\circ} \mathrm{E} 75^{\circ} \mathrm{E}$

0800 LT

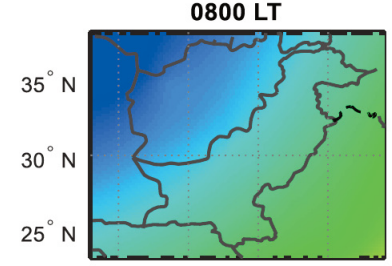

$60^{\circ}$ E $65^{\circ}$ E $70^{\circ}$ E $75^{\circ} \mathrm{E}$ 1400 LT

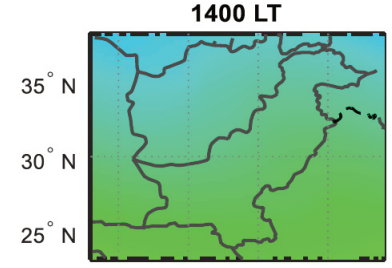

$60^{\circ} \mathrm{E} 65^{\circ} \mathrm{E} 70^{\circ} \mathrm{E} 75^{\circ} \mathrm{E}$ 2000 LT

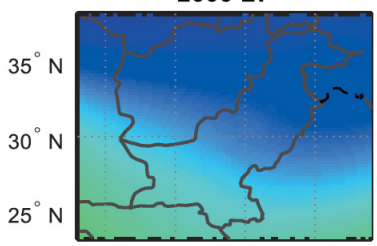

$60^{\circ}$ E $65^{\circ}$ E $70^{\circ}$ E $75^{\circ} \mathrm{E}$

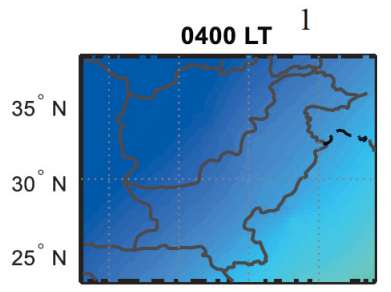

$60^{\circ} \mathrm{E} 65^{\circ} \mathrm{E} 70^{\circ} \mathrm{E} 75^{\circ} \mathrm{E}$

1000 LT

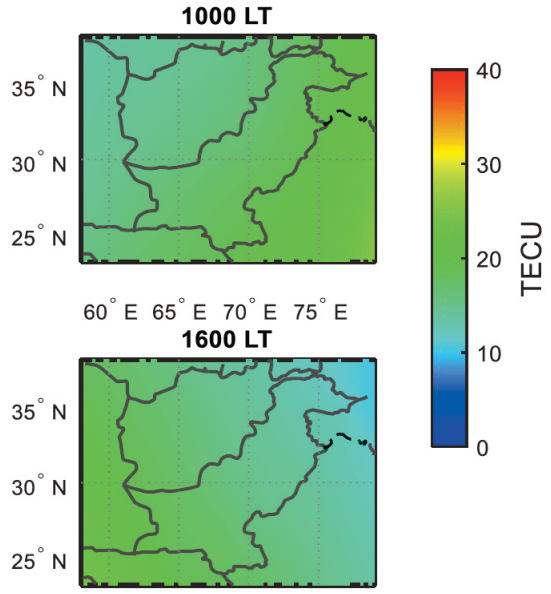

$60^{\circ} \mathrm{E} 65^{\circ} \mathrm{E} 70^{\circ} \mathrm{E} 75^{\circ} \mathrm{E}$ 2200 LT

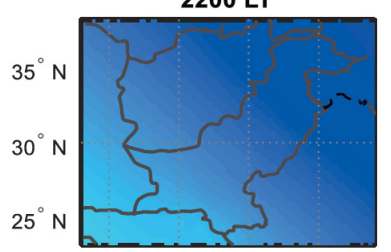

$60^{\circ} \mathrm{E} 65^{\circ} \mathrm{E} 70^{\circ} \mathrm{E} 75^{\circ} \mathrm{E}$

Figure 9. TEC maps over Pakistan by local model for DOY: 312, Year 2019.

In order to emphasize the importance of having a local TEC model for our region under consideration, we tested the performance of our model under geomagnetic disturbed conditions on Day 134, 2019. One of the many plasma clouds expelled by the sun, reached our planet on 14 $4^{\text {th }}$ May 2019 (Day 134) causing the conditions of a G3 geomagnetic storm. The global morphology for $14^{\text {th }}$ May 2019 is depicted (Figure 10). The solar wind increased from $440 \mathrm{~km} / \mathrm{s}$ to $560 \mathrm{~km} / \mathrm{s}$ by 11:00 UTC, after which it started to decrease. The Bz of IMF started a downward trend after 03:00 UTC and dipped to as low as $-12 \mathrm{nT}$ at 06:00 UTC. The z component and field intensity of the local magnetic field was obtained from the magnetic observatory installed at Sonmiani $\left(\right.$ Co - latitude $=64.8832^{\circ}$, longitude $=$ $66.4487^{\circ}$ ) with its data available at the INTERMAGNET website (https://www.intermagnet.org/).The local magnetic field, exhibited pattern similar to the IMF thus confirming the local effect of the storm. As expected the NOAA planetary $\mathrm{Kp}$ index of 6 was obtained for the 3-6 hour band and 7 for 6-9 hour band. 
The interpolated TEC values at the test location for Day 133-135 were obtained from the CODE-IONEX file, IRI2016 and local model (Figure 11). The test location was selected instead of a local GPS station, in order to avoid bias towards the local model. The analysis reveal the IRI-2016 does not exhibit any response to the occurrence of storm because the database of the storm model for IRI-2016 consists of insufficient data for storm period. The same has been concluded by a previous study of a nearby region [Reddybattula et al., 2019].The TEC data of CODE-GIM and local model for three quiet days of the month with $K p=2\left(5^{\text {th }}\right.$ May, $8^{\text {th }}$ May and $12^{\text {th }}$ May) was averaged to obtain a reference quiet value respectively. The percentage increase of the TEC for each model during storm day was calculated from its reference quiet value. It was found that GIM shows a maximum TEC enhancement of 13.8\%, whereas the local model exhibits very pronounced TEC enhancement of up to $52.7 \%$. The analysis of geomagnetic storm response is of significance as it affects the satellite-based technological and scientific applications; in this regard, local model will enable a better analysis of geomagnetic phenomenon leading to improved forecasting.
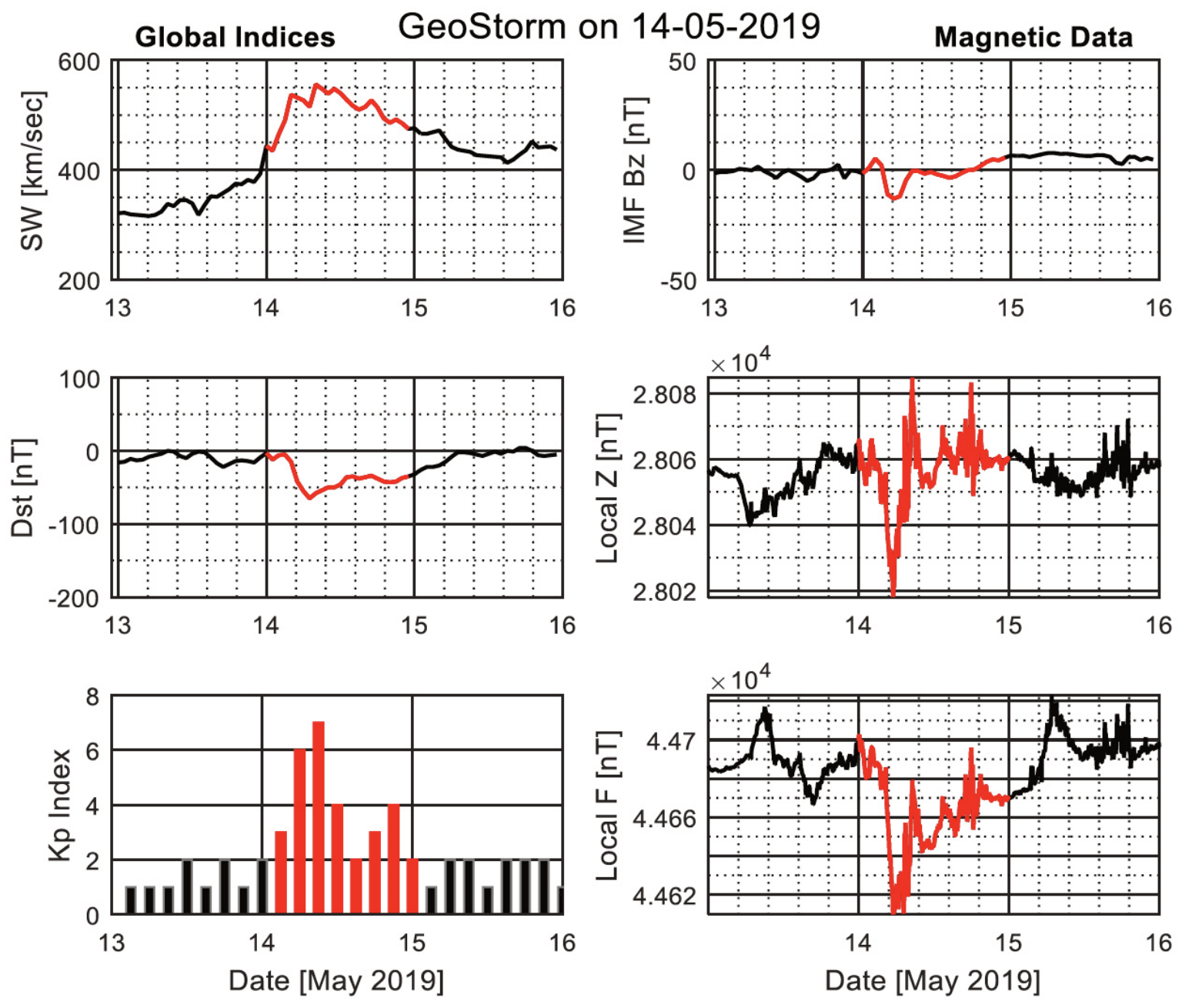

Figure 10. Global morphology and local magnetic field for G3 storm on 14th May 2019. 


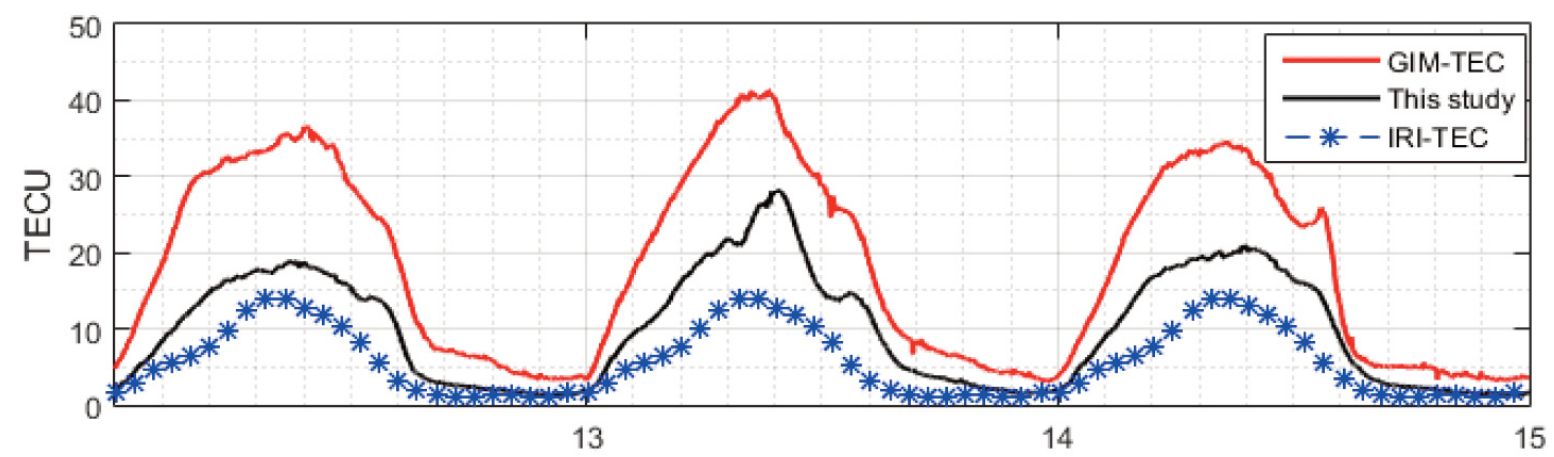

Figure 11. TEC estimates from CODE-GIM, IRI-2016 and this study at test location for Day 133-135 (2019).

\section{Conclusion}

Currently, TEC maps provided by IRI-2016 and CODE-GIM are being used for TEC variations and estimation within Pakistan. However, these models not only offer a low resolution in space and time but exhibit poor performance since no GNSS station is located within Pakistan. Due to lack of GNSS-related research and infrastructure in the region of interest no customized solution for TEC maps is currently available for this area. This study has developed a local TEC map with a resolution of latitude $0.2^{\circ} \times$ longitude $0.2^{\circ} \times 5$ mins, for the first time, over the region of Pakistan. The research has also determined that for GPS-based TEC mapping of this region the spherical harmonic function may be expanded up to 6 degrees for optimal results. The TEC values estimated by the local TEC maps are compared to the presently used TEC maps (provided by CODE-GIM and IRI-2016) as well as actual TEC measurements from a GPS scintillation receiver. It was found that the local TEC maps by this study are an accurate estimation of TEC for this region for Year 2019 as compared to CODE-GIM and IRI-2016. The study also found that the local TEC maps correlate better to CODE-GIM rather than IRI-2016. We have also analyzed the performance of all three models in case of a G3 geostorm that occurred on $14^{\text {th }}$ May 2019 and concluded that the local model exhibits much pronounced enhancements in TEC as compared to standard models.

The global models are focused on the universal distribution and climatological analysis of TEC and result in missing out small-scale local variations due to sparse distribution of stations. We have demonstrated that the regional maps are able to reproduce local variability, especially around the EIA. The TEC maps presented in this study may be utilized to accurately estimate the ionosphere delay for GPS users in Pakistan and improve the positioning solutions as well as to observe the geo storms that occur over this region. The study will contribute towards ionosphere mapping and forecasting service over Pakistan by allowing reproduction of local ionosphere morphology.

For future work, we recommend generation of local TEC maps using multi-constellation data (e.g. Galileo/ BDS). It is also suggested to explore the advantage of TEC calculation with geostationary satellite data [Savastano et al., 2019].

Acknowledgements. The authors wish to acknowledge the facilities provided by National University of Science and Technology (NUST), Institute of Space Technology (IST) and IBA Sukkur for this research. Moreover, we would like to express gratitude to the editor(s) and reviewers for their valuable comments and feedback. The results presented in this paper rely on data collected at magnetic observatories. We thank the national institutes that support them and INTERMAGNET for promoting high standards of magnetic observatory practice (www.intermagnet.org). 


\section{References}

Barrile V., M. Cacciola and F. Cotroneo (2006). Multitpath Reduction of GPS Measures through Heuritics Techniques of Compensation. Progress In Electromagnetic Research Symposium 2006, Cambridge, 528-532.

Bilitza D. (1986). International reference ionosphere: recent developments. Radio Sci., 343-346.

Bilitza D. (1990). Including auroral boundaries in the IRI model, NSSDC Report, 90-22, available from National Space Science Data Center, Greenbet, MaryLand 20771, USA.

Bilitza D. (2001). International reference ionosphere 2000, Radio Sci., 36(2), 261-275.

Bilitza D. (2018). IRI the International Standard for the Ionosphere. Advances in Radio Science, 16, 1-11.

Bilitza D. and B. Reinisch (2008). International reference ionosphere 2007: improvements and new parameters, Adv. Space Res., 42(4), 599-609.

Cannon P., M. Angling, L. Barclay, C. Curry, C. Dyer, R. Edwards, G. Greene, M. Hapgood, R. Horne, D. Jackson, C. Mitchell, J. Owen, A. Richards, C. Rogers, K Ryden, S. Saunders, M. Sweeting, R. Tanner, A. Thomson C. Underwood (2013). Extreme space weather: impacts on engineered systems and infrastructure, London: Royal Academy of Engineering. Retrieved from http://bit. ly/11OdBNN

Chapman S. (1931). The absorption and dissociative or ionizing effect of monochromatic radiation in an atmosphere on a rotating earth, Proc. Phys. Soc., 43(1), 26-45.

Davies K. (1990). Ionospheric Radio, IEEE Electromagnetic Waves, Series 3,. London: Peter Peregrinus.

Feltens J., and S. Schaer (1998). IGS Products for the Ionosphere, IGS Position, Proceedings of the IGS analysis centers workshop, Darmstadt, Germany. 225-232.

Habarulema J.B., L.-A. McKinnell and B. Opperman (2011). Regional ionospheric TEC modelling; working towards mapping Africa's Ionosphere, 2011, XXXth URSI General Assembly and Scientific Symposium. Istanbul, Turkey, 13-20.

Han D., H. Yun and C. Kee (2013). Performance Evaluation of Ionosphere Modeling Using Spherical Harmonics in the Korean Peninsula, J. Korean GNSS Society, 2(1), 59-65.

Hernández-Pajares M., J.M. Juan, J. Sanz, R. Orus, A. Garcia-Rigo, J. Feltens, A. Komjathy, S.C. Schaer, A. Krankowski (2009). The IGS VTEC maps: a reliable source of ionospheric information since 1998. J. Geod., 83, 263-275.

International GNSS Service. (2015). IGS Current Site Guidelines, Retrieved 04 05, 2019, from https://kb.igs.org/hc/enus/articles/202011433-Current-IGS-Site-Guidelines

Jin R., S. Jin and G. Feng (2012). M_DCB: Matlab code for estimating GNSS satellite and receiver differential code biases, GPS Solution, 541-548.

Jet Propulsion Laboratory (2015). Introduction to GAIM, Retrieved 05 03, 2019, from https://iono.jpl.nasa.gov/gaim/intro.html

Klobuchar J.A. (1982). Ionospheric Corrections for the Single Frequency User of the Global Positioning System. Galveston,Texas, USA.

Krankowski A., W. Kosek, L. Baran and W. Popinski (2005). Wavelet analysis and forecasting of VTEC obtained with GPS observations over European latitudes, J. Atmosph. Solar Terrestr. Phys., 1147-1156.

Krypiak-Gregorczyk A., P. Wielgosz and A. Borkowski (2017). Ionosphere Model for European Region Based on MultiGNSS Data and TPS Interpolation, Remote Sensing, 9, (12). 1221.

Liu A., Wang, N., Z. Li, K. Zhou and H. Yuan (2018). Validation of CAS’s final global ionospheric maps during different geomagnetic activities from 2015 to 2017, Results Phys., 10, 481- 486.

Liu Z.,Y. Li, F. Li and J. Guo (2018). Near real-time PPP-based monitoring of the ionosphere using dual frequency GPS/BDS/Galielo data, Adv. Space Res., 61(6), 1435-1443.

Mendillo M. (2006). Storms in the ionosphere: Patterns and processes for total electron content, Rev. Geophys., 44.

Ohashi M., Y. Sato, A. Yamada, Y. Kubo and S. Sugimoto (2015). Studies on Spherical Cap Harmonic Analysis for Japanese Regional Ionospheric Delays and its Prediction, 47th ISCIE International Symposium on Stochastic Systems Theory and Its Applications, Honolulu. 5-8.

Perez R.O. (2017). Ionospheric error contribution to GNSS single-frequency navigation at the 2014 solar maximum., J. Geod., 91, (4), 397-407.

Ping J., Y. Kono, K. Matsumoto, Y. Otsuka, A. Saito, C. Shum, K. Heki and N. Kawano (2002). Regional ionosphere map over Japanese Islands, Earth Planets Space, 54, 13-16.

Rawer K., D. Bilitza, and S. Ramakrishan (1978). International reference ionospehre, 78. Brussels, Belgium, 


\section{Maria Mehmood et al.}

International Union of Radio Science (URSI), 2, Brussels, Belgium.

Reddybattula K.D., S.K. Panda, K. Ansari and V.S. Peddi (2019). Analysis of ionospheric TEC from GPS, GIM and global ionosphere models during moderate, strong, and extreme geomagnetic storms over Indian region, Acta Astronautica, 161, 283-292.

Reza G.F., H. Pierre, D. Donald and B. David (2011). Developing a GPS TEC mapping service over Canada, Space Weather, 9.

Saito S., Sunda, S., J. Lee, S. Pullen, S. Supriadi, T. Yoshihara, M. Terkildsen, F. Lecat and ICAO APANPIRG Ionospheric Studies Task Force (2017). Ionospheric delay gradient model for GBAS in the Asia-Pacific region, GPS Solutions, 21, 1937-1947.

Savastano G., A. Komjathy, E. Shume, P. Vergados, M. Ravanelli, O. Verkhoglyadova, X. Meng, M. Crespi (2019). Advantages of Geostationary Satellites for Ionospehric Anomaly Studies: Ionospheric Plasma Depletion Following a Rocket Launch, Remote Sensing, 1734.

Sharif M.A. and S. Farzaneh (2017). The ionosphere electron density spatio-temporal modellig based on the TEC Regional modelling and prediction using ANN Slepian basis functions, Acta Geodaet. Geophys., 52(1), 5-18.

Stanislawska I., G. Juchnikowski, R. Hanbaba, H. Rothkael, G. Sole and Z. Zbyszynski (2000). COST 251 Recommended Instantaneous Mapping Model of Ionospheric Characteristics - PLES, Phys. Chem. Earth (C), 25(4), 291-294.

Subirana J.S., J.M. Zornoza and M. Hernandez-Pajares (2013). GNSS Data Processing - Volume I: Fundamentals and Algorithms, Noordwijk, The Netherlands: European Space Agency (ESA).

Sur D. and A. Paul (2013). Comparison of standard TEC models with a Neural Network based TEC model using multistation GPS TEC around the northern crest of Equatorial Ionization Anomaly in the Indian longitude sector during the low and moderate solar activity levels of the 24th sola, Adv. Space Res. 810-820.

Tariq M.A., M. Shah, M. Ulukavak and T. Iqbal (2019). Comparison of TEC from GPS and IRI-2016 model over different regions of Pakistan during 2015-2017, Adv. Space Res., 707-718.

Wielgosz P., D. Grejner-Brzezinska, and I. Kashani, I. (2003). Regional ionosphere mapping with kriging and Multiquadric methods, J. Global Posit. Sys., 2(1), 48-55.

Xin Z., J. Shuanggen, M. Cetin and F. Jialiang (2016). Evaluation of regional ionospheric grid model over China from dense GPS observations, Geod. Geodyn., 17(5), 361-368.

Yilmaz A., K. E. Akdogan and M. Gurun (2009). Regional TEC mapping using neural networks, Radio Sci., 44.

Zhao X., S. Jin, C. Mekik and J. Feng (2016). Evaluation of regional ionospheric grid model over China from dense GPS observations, Geod. Geodyn., 7(5), 361-368.

Zhizhao L. and Y. Gao, Y. (2004). Ionospheric TEC predictions over a local area GPS reference network, GPS Solutions, 23-29. 